\title{
CONTRIBUTION TO THE COINCIDENCE OF MALIGNANT TUMOURS AND SOME ALLERGIC MANIFESTATIONS
}

\author{
Pavel Rohan ${ }^{\mathrm{a}}$, Stanislav Komenda ${ }^{\mathrm{b}}$
}

\author{
a Montreal, Quebec, Canada \\ ${ }^{b}$ Institute of Medical Biophysics, Biometrics and Informatics at the Faculty of Medicine, Palacky University, Olomouc, \\ Czech Republic, e-mail: komenda@tunw.upol.cz
}

Received: June 20, 2001

Key words: Retrospective study / Malignancy / Allergy or allergy-like manifestation / Frequency of occurrence / Chi-square comparison

Clinical observations during the past decades led us to the early empirical assumptions that adult patients affected by certain types of allergic manifestations may have a lower prevalence of malignant tumors. In order to test those observations as well as some contradictory reports ${ }^{1-3}$, we conducted a retrospective study using 32 years of records and statistics of a medium-sized hospital (420 beds) at St. Mary's Hospital, a general, non-chronic, teaching hospital, affiliated with McGill University in Montreal. The study has been realized during two periods: (a) 19651989 and (b) 1990-1996.

The OBJECTIVE of this presentation has been to summarize some preliminary results concerning malignancies of patients with regard to their medical history of allergic manifestations (ALL).

The SAMPLE: Patients followed at St. Mary's Hospital (SMH). The study consisted of adult patients (15 years and over) admitted at SMH with all kinds of diagnoses. During the 32 year period (1965-1996), a total of 470,462 patients have been admitted at SMH, which represents a yearly admittance average of 14,702 patients. Our sample concerned the subtotal of 12,206 patients with malignant tumors, admitted at SMH during the 32 year period (yearly average of 381 patients), with or without a history of ALL. Patients followed at SMH in several out-patient clinics as well as those treated at home under SMH supervision, or in other SMH-connected facilities have been excluded from this survey. Repeated admissions of the same patient with malignant tumor have been considered as separate cases. From the subtotal of 12,206 admitted malignancies, 366 have been identified with both tumors and ALL, which represents $3.0 \%$ during the 32 year period (1965-1996). The remaining 11,840 patients with tumors but no recorded ALL amounted thus to $97.0 \%$ of the hospital admitted malignancies. The latest decimal WHO-classification of diseases has been used for diagnosing of both tumors and ALL. ${ }^{4}$ For the exhaustive summarization see Table 1.

From our target population of 366 patients with both tumors and ALL, we have been able to study in more details the earlier period (1965-1989) than the more recent one (1990-1996). The 366 patients represent an incidence of 11.4 cases per year. Calculations of chi-square as well as the expected and observed fre- quencies have been performed. The shortcomings and biases of our approach have been taken into consideration. ${ }^{5}$ The respective chi-square testing is equivalent with the comparison of the relative frequencies of the occurrence of ALL in tumorous and non-tumorous groups.

Some RESULTS from the first period (1965-1989): there were 187 patients with both tumors and ALL (51.1\% from the total of 366 patients (1965-1996). The sex distribution among them was quite uniform: $49.2 \%$ of men and $50.8 \%$ of women. The average age amounted to 66.4 years; it was higher among men (68.3 years) than among women (64.5 years). The distribution of age groups formed a typical bell-shaped curve, with a peak in the 60-69 age group. Smoking was not a determinant; this information was available among $72.7 \%$ of patients. Neither was the type of occupation a discriminating factor. Patients with a history of ALL concerned the following categories of tumors (in decreasing order): malignancies of the digestive system $(26 \%)$, urogenital tumors $(16 \%)$, respiratory tumors $(13 \%)$, gynecologic tumors, breast cancers, malignant hemopathies (about $10 \%$ in each category) and about $15 \%$ of other malignancies. $^{3}$

Some COMMENTS for the total 32 year period (1965-1996):

The observed value of 366 patients admitted at SMH with a confirmed malignancy, having had a recorded history of ALL, is relatively low, amounting to (see Table 1):

$3.00 \%$ of all admitted 12,206 malignancies $4.85 \%$ of all 7,554 cases with a history of ALL $0.08 \%$ of all 470,462 hospital admissions. 
Table 1. Summary cross-tabulation of the presence of malignant tumors (MT) versus allergies or allergy-like manifestations (ALL), within the period 1965-1996.

ALL

\begin{tabular}{lrrr}
\multicolumn{4}{c}{ ALL } \\
\hline MT & Yes & No & Total \\
\hline & & & \\
Yes & 366 & 11,840 & 12,206 \\
No & 7,188 & 451,068 & 458,256 \\
Total & 7,554 & 462,908 & 470,462
\end{tabular}

Chi-square $(1$ d.f. $)=153.88$, highly significant

Conditional frequencies:

$\mathrm{P}($ ALL-Yes $\mid$ MT-Yes $)=366 / 12,206=0.0300$

$\mathrm{P}($ ALL-Yes $\mid \mathrm{MT}-\mathrm{No})=7,188 / 458,256=0.0157$

Table 2. Localization of malignant tumors among the patients suffering with allergies or allergy-like manifestations (ALL), within the period 1965-1996.

\begin{tabular}{|c|c|c|}
\hline \multicolumn{3}{|c|}{ Number } \\
\hline Type of ALL & of cases & $\begin{array}{l}\text { Localization } \\
\text { of malignancies }\end{array}$ \\
\hline \multirow[t]{4}{*}{ Allergic rhinitis } & 4 & Bladder (1) \\
\hline & & Lung (1) \\
\hline & & Lymphoma (1) \\
\hline & & Rectum (1) \\
\hline \multirow[t]{2}{*}{ Urticaria } & 2 & Esophague (1) \\
\hline & & Breast (1) \\
\hline \multirow[t]{2}{*}{ Angioneuritic edema } & 3 & Rectum (1) \\
\hline & & Other (2) \\
\hline \multirow[t]{2}{*}{ Food allergy } & 0 & \\
\hline & & $\begin{array}{l}\text { In decreasing order } \\
\text { of occurrence }\end{array}$ \\
\hline Asthma & 270 & $\begin{array}{l}\text { Breast, Lung, Colon, } \\
\text { Bladder, Rectum, Stomach, } \\
\text { Prostate, Other }\end{array}$ \\
\hline Eczema & 64 & $\begin{array}{l}\text { Colon, Skin, Lung, Prostate, } \\
\text { Other }\end{array}$ \\
\hline Drug allergy & 22 & Different localizations \\
\hline Other & 1 & Unspecified \\
\hline Total & 366 & \\
\hline
\end{tabular}

Remark: Blanks mean there may be other possible tumor localizations.

Table 3. Patients with a history of allergies (Group I) or allergy-like manifestations (Group II) among those admitted with malignant tumors.

Period

\begin{tabular}{lccr}
\hline & & & \\
Group I & $1965-1989$ & $1990-1996$ & Total \\
Allergic rhinitis & 4 & 0 & 4 \\
Urticaria & 1 & 1 & 2 \\
Angioneuritic edema & 1 & 2 & 3 \\
Food allergy & 0 & 0 & 0 \\
Subtotal (I) & 6 & 3 & 9 \\
Group II & & & \\
Asthma & 98 & 173 & 271 \\
Asthma + Eczema & 1 & 0 & 1 \\
Eczema & 60 & 2 & 62 \\
Eczema + Drug allergy & 1 & 0 & 1 \\
Drug allergy & 20 & 1 & 21 \\
Other & 1 & 0 & 1 \\
Subtotal (II) & 181 & 176 & 357 \\
Total (I+II) & 187 & 179 & 366
\end{tabular}

Table 4. Cross-tabulation of the presence of malignant tumors (MT) versus allergies or allergy-like manifestations (ALL), within the period 1990-1996.

ALL

\begin{tabular}{lrrr}
\hline & & & \\
MT & Yes & No & Total \\
Yes & 179 & 3,170 & 3,349 \\
No & 2,677 & 110,898 & 113,575 \\
Total & 2,856 & 114,068 & 116,924
\end{tabular}

Chi-square $(1$ d.f. $)=180.70$, highly significant

Conditional frequencies:

$\mathrm{P}($ ALL-Yes $\mid$ MT-Yes $)=179 / 3,349=0.0534$

$\mathrm{P}($ ALL-Yes $\mid \mathrm{MT}-\mathrm{No})=2,677 / 113,575=0.0236$

Table 5. Cross-tabulation frequencies of the malignant tumors (MT) versus allergies or allergy-like manifestations (ALL), in dependence on year of evidence.

\section{Number of patients}

Year of MT ALLMT+ No MT Chi-square P(ALL-Yes|MT-Yes)

evidence only only ALL No ALL Significance P(ALL-Yes|MT-No)

\begin{tabular}{|c|c|c|c|c|c|c|}
\hline 1990 & 423 & 217 & 15 & 15,785 & $\begin{array}{r}11.67 \\
0.006, \text { h. s. }\end{array}$ & $\begin{aligned} 15 / 438 & =0.0342 \\
217 / 16,002 & =0.0136\end{aligned}$ \\
\hline \multirow[t]{2}{*}{1991} & 475 & 324 & 20 & 16,175 & 9.43 & $20 / 495=0.0404$ \\
\hline & & & & & 0.002 , h.s. & $324 / 16,499=0.0196$ \\
\hline \multirow[t]{2}{*}{1992} & 464 & 376 & 13 & 16,807 & 0.00 & $13 / 477=0.0273$ \\
\hline & & & & & 0.528 , n. s. & $376 / 17,183=0.0219$ \\
\hline \multirow[t]{2}{*}{1993} & 443 & 400 & 18 & 15,809 & 3.22 & $18 / 461=0.0390$ \\
\hline & & & & & 0.073 , n. s. & $400 / 16,209=0.0247$ \\
\hline \multirow[t]{2}{*}{1994} & 515 & 409 & 32 & 16,262 & 23.14 & $32 / 547=0.0585$ \\
\hline & & & & & 0.000 , h.s. & $409 / 16,671=0.0245$ \\
\hline \multirow[t]{2}{*}{1995} & 391 & 457 & 31 & 15,211 & 25.93 & $31 / 422=0.0735$ \\
\hline & & & & & 0.000 , h.s. & $457 / 15,668=0.0292$ \\
\hline \multirow[t]{2}{*}{1996} & 459 & 494 & 50 & 14,849 & 62.85 & $50 / 509=0.0982$ \\
\hline & & & & & 0.000 , h.s. & $494 / 15,343=0.0322$ \\
\hline Total & 3,170 & 677 & 179 & 110,898 & 120.62 & $179 / 3,349=0.0534$ \\
\hline
\end{tabular}

Table 6. Types of allergies or allergy-like manifestations (ALL) in cancerous patients, 1990-1996, in dependence on year of evidence.

\begin{tabular}{|c|c|c|}
\hline \multicolumn{2}{|c|}{$\begin{array}{ll}\text { Year of } & \text { Number of } \\
\text { evidence } & \text { ALL patients }\end{array}$} & ALL types \\
\hline 1990 & 15 & Asthma (15) \\
\hline \multirow[t]{3}{*}{1991} & 20 & Asthma (18) \\
\hline & & Eczema (1) \\
\hline & & Angioneuritic edema (1) \\
\hline 1992 & 13 & Asthma (13) \\
\hline \multirow[t]{2}{*}{1993} & 18 & Asthma (17) \\
\hline & & Drug allergy (1) \\
\hline \multirow[t]{3}{*}{1994} & 32 & Asthma (30) \\
\hline & & Urticaria (1) \\
\hline & & Angioneuritic edema (1) \\
\hline \multirow[t]{2}{*}{1995} & 31 & Asthma (30) \\
\hline & & Eczema (1) \\
\hline 1996 & 50 & Asthma (50) \\
\hline Total & 179 & \\
\hline
\end{tabular}

Comment: No allergic rhinitis nor food allergy detected. 
Some special comments are in order for the 272 cases of unspecified ASTHMA, representing 74.3\% of our target population. This high value, containing probably a considerable part of cases with a non-allergic etiology, may contribute to the excess of the observed value. These results are in accordance with the findings of some other authors ${ }^{6-8}$ about the association of asthma with lung cancer or with other malignancies. In a report, bronchogenic carcinoma could masquerade as bronchial asthma. ${ }^{9}$ Vesterinen et al. ${ }^{6}$ evaluated the elevated risk of some cancers among 77,952 asthma patients. Some differences in the immune system may, however, result in a reduced cancer risk reported in this study which linked two Finnish Registers. The prognosis, measured by a 5 year survival rate, has been found similar among asthmatic and non-asthmatic cancer patients in Finland. In another extensive Swedish study ${ }^{8}$, a cohort of 64,346 hospitalized asthma patients was followed with respect to cancer development. In general, a marked reduction of cancer incidence was found, 2 out of 3 of the expected numbers, with the exception of two cancer types: of the respiratory tract and of endocrine glands. The authors found a more noticeable reduction in cancer risks for multiple myeloma, breast cancer, uterine body tumors, and stomach cancer. The interpretation of these phenomena may prove difficult without further studies.

In our present study, the number of asthmatic patients with malignant tumors increased from the first period (1965-1989) to the recent one (1990-1996) from 99 to 173 , forming a total of 272 cases for the 32 year period.

For URTICARIA and ANGIOEDEMA cases, the values remained invariably low during the two periods, amounting to a total of 2 and 3 , respectively. The coincidence of urticaria or angioedema with the occurrence of malignancies have been studied in recent years and yielded some controversial findings. ${ }^{10-15}$

The result of 64 malignancies in patients with a history of ECZEMA may also need some comments. This diagnosis may well contain a non-negligible amount of cases with toxic or irritable skin reactions of non-allergic origin. They may also include some cutaneous manifestations to drug intolerance or side-effects to antineoplastic chemotherapy, not related to allergies. Our observations are in agreement with another author. ${ }^{16}$ During the two periods, the number of malignancies decreased from 62 to 2 . In patients with malignancies and a history of DRUG ALLERGY, we found a likewise decrease, from 21 to 1 (a total of 22). All these figures are to be seen in Table 3 .

The results found in the more recent period of 1990 to 1996 can be discussed by means of the figures presented in the following Tables 4 to 6 .

From Table 4 highly significant difference in the occurrence of ALL has been proved, when compared the groups of patients with and without malignant tumors - the association being positive.
In Table 5, the same comparison is carried out separately for each year of the period under study. With the exception of the years 1992 and 1993, a positive association between the occurrence of tumor and ALL was proved to exist, chi-square test with the Yates correction being the inductive method.

Finally, the distribution of allergies and allergy-like manifestations was given in Table 6, separately for each year of reporting.

\section{SUMMARY}

This study has been based on our long-term clinical observations as well as upon a retrospective statistical and record review of 470,462 patients admitted into a medium-sized University affiliated hospital during a 32 year period between $1965-1996$.

Among the total, 12,206 patients have been admitted for a malignant pathology $(2.6 \%)$. Their medical charts have been screened for the recorded histories of allergic manifestations. This target population amounted to 366 patients.

Our assumption about an eventual positive association of some allergies with the occurrence of malignant tumors got some support.

Besides the usual disadvantages, biases and confounding factors involved in retrospective studies, our review has an additional serious shortcoming: On the one side, we are dealing with the "hard evidence" of histologically proven malignant tumors, confronted with the "soft evidence" of allergic manifestations, without any immunological or other diagnostic confirmation, on the other side.

Even the apparent phenomenon of an existing association of certain allergies with the occurrence of malignant tumors cannot be corroborated without further relevant prospective investigations.

\section{ACKNOWLEDGEMENTS}

Special mention is in order with sincere thanks to - Mrs. Elise Laviotte, from SMH, Montreal, QC, Canada, who helped efficiently with the collection of hospital charts and yearly statistical data for this study

- Jiri (George) Rohan, MD, Brno, Czech Republic, for his help with the review of relevant bibliographical texts and summaries.

\section{PRÉCIS}

Data have been summarized within the 32 year period, enabling to evaluate the degree of coincidence of the occurrence of tumors and allergies or allergy-like manifestations. Some positive association was proved to exist. 


\section{BACKGROUND}

There exists evidence, sometimes contradictory, on the association between the occurrence of malignant tumors and allergies or allergy-like manifestations. The study is a contribution to the discussion of the problem.

\section{METHODS}

A retrospective study was carried out based on the 32 year records - review of a medium-sized hospital (420 beds) at St. Mary s Hospital, a general, non-chronic, teaching hospital, affiliated with McGill University in Montreal.

\section{RESULTS}

In the whole sample, as well as in its age delimited parts, higher occurrence of allergies or allergy-like manifestations was found among the patients with malignancies, in comparison with those without malignancies. These differences were significant and highly significant, in most cases, as documented by the chi-square testing when comparing the respective frequencies.

\section{CONCLUSIONS}

Retrospective study of this kind may be loaded by some factors inducing potential biases into the reasoning. Nevertheless, the evidence presented rejects the hypothesis of the independence of the occurrence of malignancies on one side and the allergies or allergy-like manifestations on the other side, recognizing the existence of some positive association between both events. The results derived from the data might be the reason for a more sophisticated and detailed study of the problem.

\section{REFERENCES}

1. Fisherman EW (1960) Does the allergic diathesis influence malignancy? J. Allergy 31, 74-78.

2. McKee WD, et al. (1967) A double-blind study of the comparative incidence of malignancy and allergy. J. Allergy 39, 294-301.

3. Rohan PC, Jequier JC (1990) Possible interaction of allergies with the occurrence of malignant tumors. Med. Bulletin 32, 15-22.

4. WHO International Classification of Diseases, 10th Revision (ICD-10). Geneva, WHO,1992.

5. Last JM (1986) Epidemiology and Health Information. In: MaxcyRosenau Public Health and Preventive Medicine, 12th ed., Appleton-Century-Crofts, Norwalk, Connecticut, USA.

6. Vesterinen E, et al. (1993) Cancer incidence among 78,000 asthmatic patients. Internat. J. Epidemiol. 1993, 22, 976-82.

7. Vesterinen E, et al. (1993) Survival rates in lung cancer patients with and withoutbronchial asthma. Acta Oncologica 32, 517-520.

8. Kallen B, et al. (1993) Cancer risk in asthmatic subjects selected from hospital discharge registry. Europ. Respirat. J. 6, 694-697.

9. Singh B, et al. (1995) Bronchogenic carcinoma masquerading as bronchial asthma. J. Assoc. of Physicians of India 43, 579.

10. Lindelof B, et al. (1990) Chronic urticaria and cancer: An epidemiological study of 1,155 patients. Brit. J. Dermatol. 123, 453-456.

11. Bourezane Y, et al. (1994) Urticaire chronique révelatrice d un phéochromocytome vesical. Presse méd. 23, 717.

12. Govindaraju S, et al. (1993) Urticaire chronique: évolution vers un myelome á IgM. Rév. Méd. int. 14, 780-783.

13. Lafenillade A, et al. (1993) Oedeme angioneurotique acquis au cours d'une leucémie lymphoide chronique. Presse Méd. 22, 1421.

14. Bain BJ, et al. (1993) Acquired angioedema as the presenting feature of lymphoproliferative disorders. Cancer 72, 3318-3322.

15. O'Donnell BT, Foulds IS (1993) Thyroid carcinoma presenting as angio-edema. Brit. J. Dermatol. 28, 588-589.

16. Kao NL, Richmond GW (1993) Cutaneous markers of internal malignancy. J. Amer. Assoc. Dermatol. 28, 147-164. 